\title{
CONDIÇÕES DE TRABALHO E CARACTERÍSTICAS SÓCIO- DEMOGRÁFICAS RELACIONADAS À PRESENÇA DE DISTÚRBIOS PSÍQUICOS MENORES EM TRABALHADORES DE ENFERMAGEM ${ }^{1}$
}

\author{
Ana Lúcia Cardoso Kirchhof², Tânia Solange Bosi de Souza Magnago³, Silviamar Camponogara4, Rosane Har- \\ ter Griep ${ }^{5}$, Juliana Petri Tavares 6 , Francine Cassol Prestes ${ }^{7}$, Lucilene Gama Paes ${ }^{8}$
}

${ }^{1}$ Dados da pesquisa multicêntrica “Saúde dos trabalhadores de enfermagem em hospitais públicos universitários das Regiões Sul e Sudeste do Brasil”. Apoio financeiro do CNPq.

${ }^{2}$ Doutora em Enfermagem. Pesquisadora Visitante da Universidade Federal do Paraná. Professora Aposentada da Universidade Federal de Santa Catarina. Santa Catarina, Brasil. E-mail: kirchhof@terra.com.br

${ }^{3}$ Doutora em Enfermagem. Professor Adjunto do Departamento de Enfermagem da Universidade Federal de Santa Maria (UFSM). Rio Grande do Sul, Brasil. E-mail: tmagnago@terra.com.br

${ }^{4}$ Doutora em Enfermagem. Professor Adjunto do Departamento de Enfermagem da UFSM. Rio Grande do Sul, Brasil. E-mail: silviaufsm@yahoo.com.br

${ }^{5}$ Doutora em Ciências. Pesquisadora do Laboratório de Educação Saúde e Ambiente da Fundação Osvaldo Cruz. Rio de Janeiro, Brasil. E-mail: rohgriep@terra.com.br

${ }^{6}$ Mestranda do Programa de Pós-graduação em Enfermagem da UFSM. Rio Grande do Sul, Brasil. E-mail: julianapetritavares@ yahoo.com.br

7 Acadêmica do Curso de Enfermagem da UFSM. Rio Grande do Sul, Brasil. E-mail: francassol@bol.com.br

${ }^{8}$ Enfermeira Residente do Programa de Residência Multiprofissional em Saúde da Família e Comunidade da Universidade do Planalto Catarinense. Santa Catarina, Brasil. E-mail: lucilenepaes@yahoo.com.br

RESUMO: Demandas psicológicas e controle são dimensões psicossociais que podem gerar alta exigência no trabalho da enfermagem. Este estudo avalia a associação entre demanda psicológica e controle no trabalho e ocorrência de distúrbios psíquicos menores entre trabalhadores de enfermagem. Estudo transversal incluindo 491 trabalhadores de enfermagem de um hospital universitário do Rio Grande do Sul. Para avaliação dos distúrbios psíquicos menores e das dimensões psicossociais, utilizou-se questionário das versões brasileiras do Self-Report questionnaire-20 e do Job Content Questionnaire, no período de março a setembro de 2006. A prevalência de distúrbios psíquicos menores foi $18,7 \%$. Após ajustes por potenciais confundidores, a chance de distúrbios psíquicos menores foi maior $(\mathrm{OR}=2,76$; IC95\%: 1,21-6,27) no quadrante de trabalho em alta exigência, quando comparado aos trabalhadores em trabalho de baixa exigência. Considera-se necessária uma gestão organizacional participativa que inclua os trabalhadores no processo de mudanças e melhorias do ambiente laboral, principalmente no que tange às demandas e ao controle no trabalho.

DESCRITORES: Trabalho. Ambiente de trabalho. Transtornos mentais. Saúde do trabalhador. Enfermagem.

\section{WORKING CONDITIONS AND SOCIAL-DEMOGRAPHIC CHARACTERISTICS RELATED TO THE PRESENCE OF MINOR PSYCHIC DISORDERS IN NURSING WORKERS}

\begin{abstract}
Psychological demands and control are psychosocial dimensions of work that may generate high levels of stress in nursing work. This study evaluates the association between psychological demands and control in nursing work and the occurrence of minor psychic disorders among nursing workers. This transversal study includes 491 nursing workers from a university hospital in Rio Grande do Sul, Brazil. In order to evaluate the minor psychic disorders and the psychosocial dimensions, Brazilian versions of the Self-Report questionnaire-20 and the Job Content Questionnaire were used, during the period of March to September of 2006. An $18.7 \%$ prevalence of minor psychic disorders was observed. After adjustments for potentially confusing elements, the chance for minor psychic disorders was higher (OR=2.76; IC 95\%: 1.21-6.27) in the high demand working quadrant when compared to workers in the low demand working quadrant. We consider it necessary to have organizational and participatory management which includes the workers in the process of changes and improvements in the working environment, mainly those that affect demands and control in work.

DESCRIPTORS: Work. Working environment. Mental disorders. Occupational health. Nursing.
\end{abstract}




\title{
CONDICIONES DE TRABAJO Y CARACTERÍSTICAS SOCIODEMOGRÁFICAS RELACIONADAS A LA PRESENCIA DE DISTURBIOS PSÍQUICOS MENORES EN TRABAJADORES DE ENFERMERÍA
}

\begin{abstract}
RESUMEN: Las demandas psicológicas y el control son dimensiones psicosociales del trabajo que pueden generar alta exigencia en el trabajo de la enfermería. Este estudio evalúa la asociación entre la demanda psicológica y el control en el trabajo y la ocurrencias de disturbios psíquicos menores entre trabajadores de enfermería. Estudio transversal, que incluye 491 trabajadores de enfermería de un hospital universitario de Rio Grande do Sul. Para la evaluación de los disturbios psíquicos menores y de las dimensiones psicosociales, se utilizó el cuestionario de las versiones brasileñas del Self-Report questionnaire-20 y del Job Content Questionnaire, en el período de marzo a septiembre de 2006. La prevalencia de disturbios psíquicos menores fue de 18,7\%. Después de ajustes por potenciales confundidores, los casos de disturbios psíquicos menores fueron mayores (OR=2,76; IC 95\%: 1,21-6,27) en el cuadrante de trabajo en alta exigencia, comparados a los trabajadores en trabajo de baja exigencia. Se considera necesaria una gestión organizacional participativa que incluya a los trabajadores en el proceso de cambios y mejoras del ambiente laboral, principalmente en lo que se refiere a las demandas y al control en el trabajo.
\end{abstract}

DESCRIPTORES: Trabajo. Ambiente de trabajo. Trastornos mentales. Salud laboral. Enfermería.

\section{INTRODUÇÃO}

O trabalho é uma atividade na qual aspectos físicos e psíquicos estão diretamente relacionados e podem tanto representar equilíbrio, desenvolvimento e satisfação, quanto podem causar tensão, desajuste e, conseqüente, adoecimento do trabalhador. ${ }^{1}$

Nas últimas décadas, dadas as transformações nos processos produtivos, as relações entre trabalho, estresse e suas repercussões sobre a saúde mental dos trabalhadores têm sido abordados em estudos com abordagens metodológicas diferenciadas. ${ }^{2-5}$ Questões como a produtividade, os acidentes de trabalho, o absenteísmo e os crescentes índices de sintomas psíquicos entre os trabalhadores de determinadas categorias profissionais têm sido objetos desses estudos. Dentre estas categorias profissionais, destacamse os trabalhadores da saúde, especialmente os atuantes em ambiente hospitalar, tendo em vista as inúmeras circunstâncias desgastantes presentes em seu cotidiano laboral. Assim, a insalubridade ou a penosidade do trabalho hospitalar, provém da permanente exposição a um ou mais fatores que produzam doenças ou sofrimento, decorrentes da própria natureza do trabalho e de sua organização, que são evidenciados por sinais e sintomas orgânicos e psíquicos inespecíficos. ${ }^{1}$

Nessa perspectiva, em estudo realizado sobre a saúde mental dos profissionais da área da saúde, há a descrição de um panorama psicodinâmico bastante complexo em que questões somadas ao estresse estritamente profissional, como a dupla jornada de trabalho, a desvalorização do trabalho feminino e a hegemonia do discurso médico em relação aos demais profissionais da área repercutem, negativamente, na saúde mental dos trabalhadores. ${ }^{6}$

Dentre os trabalhadores da saúde, estudos apontam a Enfermagem como uma das ocupações com alto risco de desgaste e adoecimento. ${ }^{7-11} \mathrm{O}$ ambiente hospitalar pode proporcionar estresse e agravos psíquicos neste trabalhador, pois é no ambiente laboral que se estabelecem as demandas de tarefas e nele o profissional experimenta variados graus de controle sobre as atividades que executa. ${ }^{3}$ Assim, o estresse laboral é resultado do desequilíbrio entre as demandas que o exercício profissional exige e a capacidade de enfrentamento do trabalhador, ${ }^{12}$ podendo resultar em sintomas de burnout. ${ }^{4}$

Diante da ausência na Classificação Internacional das Doenças de um grupo de diagnósticos de distúrbios psíquicos relacionados com o trabalho e das diferentes classificações existentes: Morbidade Psiquiátrica Menor (MPM), Transtornos Mentais Comuns (TMCs), Problemas Psiquiátricos Menores (PPMs) e Distúrbios Psiquiátricos Menores (DPMs), utilizadas para designar quadros com sintomas ansiosos, depressivos ou somatoformes e que não satisfazem a todos os critérios de doença mental, ${ }^{13}$ optou-se, neste estudo, pela terminologia DPMs. Os DPMs ganham proporção cada vez maior entre os trabalhadores de enfermagem, caracterizando-se como um problema de saúde pública. ${ }^{8-9,11}$ Tristeza, ansiedade, fadiga, diminuição da concentração, preocupação somática, irritabilidade e insônia são alguns dos sintomas relatados por indivíduos com DPMs, os quais causam incapacidade funcional comparável ou até pior que quadros crônicos já bem estabelecidos. ${ }^{14}$ 
Para entendimento destes distúrbios, estão sendo analisadas as dimensões psicossociais no contexto laboral (demandas do trabalho e controle do trabalhador sobre as atividades). ${ }^{5,8-9,11} \mathrm{No}$ ano de 1979, foi proposto o Modelo bidimensional Demanda-Controle (MDC) - Demand-Control Mode ou Job Strain que, além das demandas psicológicas do trabalho, considera o controle do trabalhador sobre as atividades laborais na análise dos processos de produção de estresse. ${ }^{3-8}$

Essas duas dimensões psicossociais abarcam aspectos específicos do processo de trabalho. $\mathrm{O}$ controle no trabalho engloba questões referentes ao uso de habilidades (o grau pelo qual o trabalho envolve aprendizagem de coisas novas, repetitividade, criatividade, tarefas variadas e o desenvolvimento de habilidades especiais individuais) e autoridade de decisão (inclui a habilidade individual para a tomada de decisões sobre o próprio trabalho, a influência no grupo de trabalho e na política gerencial). Já, a demanda psicológica diz respeito às exigências psicológicas enfrentadas pelo trabalhador durante a execução das suas tarefas. Tal demanda envolve "[...] pressão do tempo (proporção do tempo de trabalho realizado sob tal pressão), nível de concentração requerida, interrupção das tarefas e necessidade de se esperar pelas atividades realizadas por outros trabalhadores". $3: 31$

O MDC diferencia quatro tipos básicos de experiências no trabalho, formados pela interação dos níveis de demanda psicológica e de controle: alta exigência (alta demanda e baixo controle), baixa exigência (baixa demanda e alto controle), trabalho passivo (baixa demanda e baixo controle) e trabalho ativo (alta demanda e alto controle). ${ }^{3}$ Dentre as quatro situações, o trabalho em alta exigência é o que apresenta maior propensão para adoecimento físico e psicológico. Os trabalhos ativo e passivo representam risco intermediário de adoecimento, já o trabalho em baixa exigência, representa o menor risco. Este último é uma condição ideal e serve como categoria de referência durante as análises.

Tendo como base as considerações acima descritas, o presente estudo tem por objetivos verificar a prevalência de DPM nos trabalhadores de enfermagem do Hospital Universitário de Santa Maria-RS, avaliando sua associação com demanda psicológica e controle sobre o trabalho.

\section{MÉTODO}

Esta investigação, do tipo transversal, se insere em um estudo multicêntrico com traba- lhadores de enfermagem de quatro hospitais universitários públicos das regiões Sul e Sudeste do Brasil. As análises apresentadas neste artigo são baseadas nas informações obtidas de um dos hospitais participantes, o Hospital Universitário de Santa Maria. Foram definidos como elegíveis todos os 528 trabalhadores de enfermagem concursados e atuantes neste hospital.

A coleta dos dados foi realizada por um grupo de nove entrevistadoras, todas acadêmicas de enfermagem, previamente capacitadas. Utilizou-se um questionário-entrevista com questões fechadas, referentes à exposição (dimensões psicossociais do trabalho: grupos demanda-controle), ao desfecho (DPM) e a co-variáveis sócio-demográficas e laborais. As entrevistas foram realizadas individualmente, com duração de 40 a 80 minutos, em local que mantivesse a privacidade do entrevistado, no período de março a setembro de 2006.

A exposição às dimensões psicossociais do trabalho (variável independente) foi avaliada através da escala do Job Content Questionnaire (JQC), ${ }^{15}$ traduzida e adaptada para o português, ${ }^{16}$ disponível no site http//www.jcqcenter.org. Neste estudo, foram utilizadas cinco questões para avaliar demanda psicológica e nove questões para o controle sobre o trabalho. Quatro opções de resposta para as dimensões de demanda e de controle foram apresentadas, variando em discordo fortemente, discordo, concordo ou concordo fortemente. Para a composição dos grupos do MDC foram utilizados os critérios recomendados no manual do JCQ. ${ }^{15}$ Para dicotomizar as variáveis demanda e controle usou-se a mediana como ponto de corte. A partir destas duas dimensões dicotomizadas em alto e baixo, foram constituídas as quatro categorias: baixa exigência (alto controle e baixa demanda; categoria de referência), trabalho ativo (alto controle e alta demanda), trabalho passivo (baixo controle e baixa demanda) e alta exigência (baixo controle e alta demanda). Os coeficientes Alpha de Crombach para demanda psicológica e controle foram, respectivamente, 0,70 e 0,59.

O desfecho - DPM (variável dependente) - foi avaliado de acordo com escores obtidos no Self-Report Questionnaire-20 (SRQ-20), validado no Brasil. ${ }^{17} \mathrm{O}$ ponto de corte para suspeição de DPM foi de seis ou mais respostas positivas para homens e oito ou mais para mulheres. ${ }^{17}$

Outras características analisadas foram: a) variáveis sócio-demográficas: sexo; idade (tercis); escolaridade (graduado e não graduado); situação conjugal (solteiro/sem companheiro e casado/ 
com companheiro) e renda familiar per capita em salários mínimos (tercis), b) variáveis laborais: função (enfermeiro; técnico/auxiliar de enfermagem); setor; turno (diurno e noturno); carga horária semanal e outro emprego.

Para a inserção dos dados foi utilizado o programa Epi-info®, versão 6,0, com dupla digitação independente. Após a verificação de erros e inconsistências, a análise dos dados foi realizada no programa SPSS $₫ 13.0$ for windows. Foram realizadas análises univariadas e bivariadas para fins de verificação de associação entre alta exigência e DPM com cada uma das co-variáveis estudadas. $\mathrm{O}$ teste qui-quadrado foi utilizado para verificar se as associações encontradas apresentavam significância estatística $(p<0,05)$. Para a seleção das possíveis variáveis de confusão, adotou-se o critério de $\mathrm{p}<0,10$, sendo que aquelas associadas tanto à exposição quanto ao desfecho foram testadas nos modelos multivariados.

Participantes com atividades classificadas como de alta exigência, trabalho passivo e trabalho ativo foram comparados com aqueles alocados na categoria baixa exigência. A medida de associação utilizada foi a Odds Ratio (OR) e seus respectivos intervalos de confiança.

No que tange às questões éticas, a pesquisa não envolveu riscos físicos, sociais ou legais aos participantes. Os dados foram analisados somente de forma agregada, sem individualizações. A participação na pesquisa foi voluntária, tendo sido esclarecido aos trabalhadores que nenhum tipo de penalidade seria aplicada aos que não desejassem participar. Após esclarecimentos sobre a pesquisa, foi solicitada a assinatura do Termo de Consentimento Livre e Informado para Participação em Pesquisa Científica. O projeto foi aprovado pelo Parecer No 24/05 da Comissão de Ética em Pesquisa da Universidade Federal de Santa Catarina.

\section{RESULTADOS}

Participaram de estudo 491 trabalhadores, registrando-se uma perda de $7 \%$. O perfil sócio-demográfico dos trabalhadores estudados mostra predominância do sexo feminino $(88,4 \%)$, a maioria $(36,7 \%)$ com idade entre 22 e 38 anos (média 41 anos; desvio padrão (DP) = 8,7anos; idade mínima 22 e máxima 67 anos). Os graduados somavam $39,3 \%$, grande parte $(65,8 \%)$ possuía relação conjugal estável. Com relação à renda familiar per capita, 41,3\% dos trabalhadores foram classificados como percebendo menos de dois salários mínimos. Uma pequena parcela de trabalhadores é fumante $(10,8 \%)$.

Em relação ao perfil laboral do total de trabalhadores, $29,7 \%$ são enfermeiros, 32,8\% são técnicos de enfermagem e 37,5\% são auxiliares de enfermagem. Trabalham na função atual, em média, há 14,4 anos ( $\mathrm{DP}=8,3$ anos). Os setores que mais concentraram trabalhadores são as Unidades de Clínicas (Internação clínica, Internação cirúrgica, Hematooncologia e psiquiatria), Terapia Intensiva (adulto, pediátrica e neonatal) com 19,1\%, Materno infantil com $14,1 \%$, seguidas pelas Unidades Cirúrgica e de Urgência e Emergência com, respectivamente, 11,8 e $11,4 \%$ dos trabalhadores. No setor atual trabalham em média há oito anos. Os turnos diurno e noturno estão representados, respectivamente, por $59,3 \%$ e $40,7 \%$ dos trabalhadores. Do total de trabalhadores, 53\% desenvolvem uma carga horária de 36 horas semanais e $26,6 \%$ responderam possuir outro vínculo de trabalho.

A distribuição dos trabalhadores nos quatro grupos do MDC ficou da seguinte forma: 20,4\% em baixa exigência; $21,2 \%$ em alta exigência; $28,5 \%$ em trabalho ativo e $29,9 \%$ em trabalho passivo. A prevalência global de DPM nos trabalhadores de enfermagem foi $18,7 \%$. Ao se avaliar o percentual de DPM entre os trabalhadores segundo os grupos do MDC, observou-se o dobro de DPM $(27,9 \%)$ entre trabalhadores do grupo alta exigência quando comparados aos de baixa exigência (11\%). Trabalhadores dos grupos trabalho passivo e trabalho ativo obtiveram percentuais similares (em torno de $18 \%$ ).

A Tabela 1 descreve a distribuição dos trabalhadores por grupo demanda-controle segundo variáveis sócio-demográficas.

Observou-se que apenas o sexo e a situação conjugal não se mostraram associados estatisticamente às categorias avaliadas $(p>0,05)$. No que se refere às faixas etárias, observou-se freqüências mais elevadas de jovens nas categorias trabalho ativo e alta exigência. Entre não graduados e os de renda per capita mais baixa identificou-se maior freqüência de trabalhadores de enfermagem nas categorias trabalho passivo e alta exigência.

Pode-se também observar na Tabela 1, a prevalência de DPM nos trabalhadores de enfermagem segundo características sócio-demográficas. Assim, identificaram-se freqüências mais elevadas de DPM entre os trabalhadores com idade entre 22 a 38 anos $(24,4 \%)$ e entre os de renda familiar per capita menor de dois salários mínimos $(24,6 \%)$. Não se identificou associação entre as demais características e os DPM ( $p>0,05)$. 
Tabela 1 - Características sócio-demográficas dos trabalhadores de enfermagem por grupo demandacontrole e distúrbio psíquico menor $(\mathrm{n}=491)$. Santa Maria-RS, 2006

\begin{tabular}{|c|c|c|c|c|c|c|c|c|c|c|}
\hline \multirow[t]{2}{*}{ Variáveis sócio-demográficas } & \multicolumn{2}{|c|}{$\begin{array}{c}\text { Baixa } \\
\text { exigência* }\end{array}$} & \multicolumn{2}{|c|}{$\begin{array}{l}\text { Trabalho } \\
\text { passivo }\end{array}$} & \multicolumn{2}{|c|}{$\begin{array}{c}\text { Trabalho } \\
\text { ativo }\end{array}$} & \multicolumn{2}{|c|}{$\begin{array}{c}\text { Alta } \\
\text { exigência }\end{array}$} & \multicolumn{2}{|c|}{$\mathrm{DPM}^{\ddagger}$} \\
\hline & $\mathrm{n}$ & $\%$ & $n$ & $\%$ & $\mathbf{n}$ & $\%$ & $\mathbf{n}$ & $\%$ & $\mathrm{n}$ & $\%$ \\
\hline \multicolumn{11}{|l|}{ Sexo } \\
\hline Masculino & 13 & 22,8 & 16 & 28,1 & 17 & 29,8 & 11 & 19,3 & 12 & 21,1 \\
\hline Feminino & 87 & 20,0 & 131 & 30,2 & 123 & 28,3 & 93 & 21,4 & 80 & 18,4 \\
\hline \multicolumn{11}{|l|}{ Faixa etária } \\
\hline 22 a 38 anos & 33 & $18,3^{+}$ & 38 & 21,1 & 60 & 33,3 & 49 & 27,2 & 44 & $24,4^{\dagger}$ \\
\hline 39 a 46 anos & 38 & 22,4 & 63 & 37,1 & 46 & 27,1 & 23 & 13,5 & 21 & 12,4 \\
\hline 47 e mais & 29 & 20,6 & 46 & 32,6 & 34 & 24,1 & 32 & 22,7 & 27 & 19,1 \\
\hline \multicolumn{11}{|l|}{ Situação conjugal } \\
\hline Casado/companheiro & 60 & 18,6 & 88 & 27,2 & 101 & 31,3 & 74 & 22,9 & 64 & 19,8 \\
\hline $\begin{array}{l}\text { Solteiro/sem companheiro } \\
\text { Fscolaridade }\end{array}$ & 40 & 25,0 & 59 & 35,1 & 39 & 23,2 & 30 & 17,9 & 28 & 16,7 \\
\hline Não graduado & 54 & $18,1^{+}$ & 106 & 35,6 & 60 & 20,1 & 78 & 26,2 & 61 & 20,5 \\
\hline Graduado & 46 & 23,8 & 41 & 21,2 & 80 & 41,5 & 26 & 13,5 & 31 & 16,1 \\
\hline \multicolumn{11}{|l|}{ Renda familiar per capita } \\
\hline$<2$ salários mínimos & 33 & $16,3^{\dagger}$ & 74 & 36,5 & 45 & 22,2 & 51 & 25,1 & 50 & $24,6^{\dagger}$ \\
\hline 2 a 3 salários mínimos & 30 & 21,3 & 43 & 30,5 & 44 & 31,2 & 24 & 17,0 & 14 & 9,9 \\
\hline > 3 salários mínimos & 36 & 26,3 & 27 & 19,7 & 46 & 33,6 & 28 & 20,4 & 26 & 19,0 \\
\hline
\end{tabular}

* Categoria de referência, ${ }^{\dagger} \mathrm{p}<0,05,{ }^{\ddagger}$ DPM: Distúrbios psíquicos menores.

A distribuição dos trabalhadores nos grupos, ferenças expressivas entre os grupos do MDC em segundo as características laborais, demonstra di- relação a todas as variáveis analisadas (Tabela 2).

Tabela 2 - Características laborais dos trabalhadores de enfermagem por grupo demanda-controle e distúrbio psíquico menor $(\mathrm{n}=491)$. Santa Maria-RS, 2006

\begin{tabular}{|c|c|c|c|c|c|c|c|c|c|c|}
\hline \multirow[t]{2}{*}{ Variáveis laborais } & \multicolumn{2}{|c|}{$\begin{array}{c}\text { Baixa } \\
\text { exigência* }\end{array}$} & \multicolumn{2}{|c|}{$\begin{array}{c}\text { Trabalho } \\
\text { passivo }\end{array}$} & \multicolumn{2}{|c|}{$\begin{array}{c}\text { Trabalho } \\
\text { ativo }\end{array}$} & \multicolumn{2}{|c|}{$\begin{array}{c}\text { Alta } \\
\text { exigência }\end{array}$} & \multicolumn{2}{|c|}{$\mathrm{DPM}^{\S}$} \\
\hline & $\mathrm{n}$ & $\%$ & $\mathbf{n}$ & $\%$ & $\mathbf{n}$ & $\%$ & $\mathbf{n}$ & $\%$ & $\mathbf{n}$ & $\%$ \\
\hline \multicolumn{11}{|l|}{ Função } \\
\hline Enfermeiro & 36 & $24,7^{\dagger}$ & 20 & 13,7 & 74 & 50,7 & 16 & 11,0 & 23 & 15,8 \\
\hline \multicolumn{10}{|l|}{ Setor/unidade } & 20,0 \\
\hline Terapia Intensiva & 15 & $16,0^{\dagger}$ & 18 & 19,1 & 36 & 38,3 & 25 & 26,6 & 17 & 18,1 \\
\hline Clínica & 40 & 25,0 & 45 & 28,1 & 41 & 25,6 & 34 & 21,3 & 33 & 20,6 \\
\hline Cirúrgica & 14 & 24,1 & 19 & 32,8 & 9 & 15,5 & 16 & 27,6 & 10 & 17,2 \\
\hline Urgência/Emergência & 7 & 12,5 & 5 & 8,9 & 30 & 53,6 & 14 & 25,0 & 11 & 19,5 \\
\hline Ambulatorial & 13 & 32,5 & 16 & 40,0 & 4 & 10,0 & 7 & 17,5 & 9 & 22,5 \\
\hline Materno-infantil & 7 & 10,1 & 40 & 58,0 & 14 & 20,3 & 8 & 11,6 & 12 & 17,4 \\
\hline Direção e Coordenação & 4 & 28,6 & 4 & 28,6 & 6 & 42,9 & - & - & - & - \\
\hline \multicolumn{11}{|l|}{ Turno } \\
\hline Diurno & 59 & $20,3^{\dagger}$ & 80 & 27,5 & 96 & 33,0 & 56 & 19,2 & 45 & 15,5 \\
\hline Noturno & 41 & 20,5 & 67 & 33,5 & 44 & 22,0 & 48 & 24,0 & 47 & $23,5^{\dagger}$ \\
\hline \multicolumn{11}{|l|}{ Carga horária } \\
\hline $30 \mathrm{~h} / \mathrm{sem}$ & 38 & $21,1^{\dagger}$ & 64 & 35,6 & 37 & 20,6 & 41 & 22,8 & 40 & 22,2 \\
\hline $36 \mathrm{~h} / \mathrm{sem}$ & 58 & 22,3 & 67 & 25,8 & 87 & 33,5 & 48 & 18,5 & 39 & 15,0 \\
\hline $40 \mathrm{~h} / \mathrm{sem}$ & 4 & 7,8 & 16 & 31,4 & 16 & 31,4 & 15 & 29,4 & 13 & $25,5^{\ddagger}$ \\
\hline \multicolumn{11}{|l|}{ Outro emprego } \\
\hline Não & 66 & $18,2^{\dagger}$ & 115 & 31,8 & 97 & 26,8 & 84 & 23,2 & 70 & 19,3 \\
\hline Sim & 34 & 26,4 & 32 & 24,8 & 43 & 33,3 & 20 & 15,5 & 22 & 17,1 \\
\hline \multicolumn{11}{|l|}{ Demanda física } \\
\hline Menor & 72 & $26,3^{\dagger}$ & 118 & 43,1 & 53 & 19,3 & 31 & 11,3 & 42 & 15,3 \\
\hline Maior & 28 & 12,9 & 29 & 13,4 & 87 & 40,1 & 73 & 33,6 & 50 & $23,0^{+}$ \\
\hline
\end{tabular}


Entre os enfermeiros, identificaram-se freqüências mais elevadas de trabalho ativo seguido de baixa exigência enquanto que entre os técnicos as maiores freqüências foram de trabalho passivo, seguido de alta exigência. Observou-se ainda, que de acordo com o setor as freqüências mais elevadas de trabalho passivo foram identificadas entre os trabalhadores dos setores unidade cirúrgica, ambulatorial e materno-infantil, enquanto que o trabalho ativo foi mais freqüente entre a unidade de terapia intensiva, unidade clínica, emergência e direção e coordenação de enfermagem. O turno diurno apresentou freqüências mais altas de trabalho ativo, no entanto, no noturno destacou-se o trabalho passivo seguido da alta exigência. Os trabalhadores de enfermagem que referiram carga horária de trabalho mais elevada, entre aqueles que não referiram outro emprego e entre aqueles de maior demanda física identificou-se freqüências mais elevadas de classificação de alta exigência.

Segundo características laborais, os trabalhadores do noturno e os que referiram maior demanda física no trabalho apresentaram maior percentual de DPM, respectivamente, 23,5\% e $23,0 \%$. Observou-se que função, setor, carga horária e outro emprego não se mostraram associados estatisticamente à DPM $(p>0,05)$ (Tabela 2$)$.

$\mathrm{Na}$ análise individual de cada componente do MDC, houve associação positiva apenas entre DPM e baixo controle (OR=1,63; IC95\%: 1,00-2,65; $\mathrm{p}=0,037)$; a associação entre demanda psicológica e DPM não foi identificada $(\mathrm{OR}=1,56$; IC95\%:0,96-12,54; $\mathrm{p}=0,055)$.

As análises bruta e ajustada entre os grupos demanda-controle e o DPM encontram-se descrita na Tabela 3.

Tabela 3 - Associação entre dimensões psicossociais do trabalho (demanda e controle) e distúrbios psíquicos menores em trabalhadores de enfermagem. Santa Maria-RS, 2006

\begin{tabular}{lrr}
\hline Variáveis & OR $^{*}$ & IC $^{95 \%^{\dagger}}$ \\
\hline Modelo 1: dimensões psicossociais do trabalho (demanda-controle) & 2,05 & $0,94-4,46$ \\
Trabalho passivo & 1,92 & $0,87-4,23$ \\
Trabalho ativo & 3,42 & $1,59-7,62$ \\
Alta exigência & \\
Modelo 2: demanda-controle + idade & 2,09 & $0,96-4,56$ \\
Trabalho passivo & 1,88 & $0,85-4,14$ \\
Trabalho ativo & 3,42 & $1,56-7,49$ \\
Alta exigência & \\
Modelo 3: demanda-controle + idade + renda per capita familiar & 1,96 & $0,89-4,31$ \\
Trabalho passivo & 1,88 & $0,85-4,14$ \\
Trabalho ativo & 3,29 & $1,50-7,23$ \\
Alta exigência & \\
Modelo 4: demanda-controle + idade + renda per capita familiar + turno & 1,94 & $0,88-4,27$ \\
Trabalho passivo & 1,97 & $0,89-4,36$ \\
Trabalho ativo & 3,25 & $1,47-7,15$ \\
Alta exigência & \\
Modelo 5: demanda-controle + idade + renda per capita familiar + turno + carga horária & \\
Trabalho passivo & 1,89 & $0,86-4,17$ \\
Trabalho ativo & 1,91 & $0,86-4,24$ \\
Alta exigência & 3,11 & $1,41-6,88$ \\
Modelo 6: demanda-controle + idade + renda per capita familiar + turno + carga horária + & \\
demanda física & \\
Trabalho passivo & 1,95 & $0,88-4,31$ \\
Trabalho ativo & 1,71 & $0,75-3,89$ \\
Alta exigência & 2,76 & $1,21-6,27$ \\
\hline
\end{tabular}

${ }^{*} \mathrm{OR}=$ Odds Ratio, ${ }^{\mathrm{I}} \mathrm{IC}=$ intervalo de confiança

A análise de regressão logística revelou que os trabalhadores de alta exigência tiveram chance 2,76 vezes mais elevada (IC95\% $=1,21-6,27$ ) de serem classificados com DPM do que os trabalha- dores de baixa exigência, mesmo após o ajuste por variáveis identificadas como potenciais confundidoras (idade, renda per capita, turno, carga horária e demanda física). 


\section{DISCUSSÃO}

Cabe inicialmente lembrar que a realidade do mundo do trabalho é mais complexa do que os resultados alcançados por um estudo. No entanto, considera-se que dentro das limitações intrínsecas ao estudo transversal, no qual não é possível concluir a respeito de relações causais, os resultados observados contribuem para reforçar alguns achados de outros estudos, consolidando o referencial teórico adotado.

O presente estudo confirmou a associação positiva entre trabalho em alta exigência e DPM. Trabalhadores de enfermagem expostos, simultaneamente, a altas demandas psicológicas e a baixo controle no trabalho (alta exigência) apresentaram chances duas vezes maiores $(\mathrm{OR}=2,76$; IC95\%=1,21-6,27) de ocorrência de DPM do que aqueles não expostos (baixa exigência). Estudos anteriores, com população semelhante a esta, no estado da Bahia e do Rio de Janeiro, encontraram prevalência maior $(33,3 \%$ e $23,6 \%$, respectivamente) de DPM do que a detectada neste estudo $(18,7 \%) .{ }^{8,11}$ A diferença percentual entre os achados é ainda mais expressiva ao compararmos com a prevalência de agravos a saúde mental entre trabalhadores de enfermagem de unidades críticas de um hospital do Rio Grande do Sul, que foi $42,5 \% .{ }^{9}$ Essas diferenças podem estar, em parte, relacionadas às especificidades das demandas dos setores de trabalho, uma vez que naquele estudo os setores trabalham com pacientes considerados críticos, apresentando $44 \%$ dos trabalhadores em trabalho de alta exigência, enquanto neste estudo o trabalho de alta exigência ficou em $21 \%$. Logo, o trabalho de alta exigência pode estar determinando esses resultados diferenciados, haja vista que as dimensões psicossociais propostas no MDC apresentaram-se relevantes no desenvolvimento dos DPM. Este resultado é consistente com outros estudos que evidenciaram associação entre alta demanda e baixo controle no trabalho e distúrbios psíquicos. ${ }^{11-16}$

Tais achados parecem corroborar a principal hipótese do modelo de Karasek de que o trabalho realizado em condições de alta exigência é danoso à saúde dos trabalhadores, sendo considerado preditor da maioria dos sinais e sintomas adversos produzidos pelo trabalho. ${ }^{3}$

Semelhantemente aos resultados deste estudo, outros autores, identificaram associação entre alta exigência e DPM $(\mathrm{RP}=2,60$; $\mathrm{OR}=2,84$, respectivamente). ${ }^{11-16}$ No entanto, a associação positiva de DPM com trabalho ativo identificada naqueles estudos não foi observada nos nossos resultados. O alto controle parece não ter reduzido os efeitos danosos da alta demanda sobre a saúde mental dos trabalhadores pesquisados nesses estudos, tal como aponta o MDC.

A constatação de maior prevalência de DPM entre os mais jovens já foi identificada também por outros autores. ${ }^{2,11,18}$ Talvez uma explicação para este fato seja que a maior experiência no trabalho e o melhor conhecimento do contexto ajude os trabalhadores mais experientes no enfrentamento das dificuldades encontradas.

Assim como em outros estudos, percebeu-se a influência da renda na saúde mental dos trabalhadores, pois quanto menor a renda mais elevada a prevalência de DPM entre os trabalhadores..$^{10,18-19}$ Nesse sentido, estudo realizado acerca dos fatores de motivação e insatisfação no trabalho do enfermeiro, constatou que $84,6 \%$ da população estava insatisfeita com seu salário e a remuneração foi um dos fatores mais citados de insatisfação. ${ }^{20}$ Os autores ainda discorrem sobre o caráter fundamental da remuneração, inclusive como forma de valorização profissional.

Muitos trabalhadores como forma de complementar a renda assumem outros empregos. $\mathrm{O}$ acúmulo de vínculos parece ter dupla face: ser causador de prazer à medida que ofereça um melhor aporte financeiro e, por conseqüência, uma melhor qualidade de vida ou, ser causador de aumento das cargas do trabalho. ${ }^{9}$ Segundo este e outros estudos, ter outro emprego não esteve associado à DPM. Configura-se assim uma lacuna que merece ser investigada mais profundamente.

Em relação às características laborais, quando avaliado o turno de trabalho, observou-se maior prevalência de DPM nos trabalhadores do noturno. A maior parte deles encontrava-se nos grupos de trabalho passivo e alta exigência, divergindo de outros estudos.-11 Conforme classificação do MDC, os trabalhadores destes dois grupos estão associados, predominantemente ao baixo controle no trabalho. Corrobora, neste sentido, a afirmação dos autores do $\mathrm{MDC}^{3}$ de que baixos níveis de controle sobre o planejamento e a execução das atividades laborais influenciam, nocivamente, a saúde dos trabalhadores. Ou seja, ausência ou pouco controle pode ter efeitos na auto-estima, ser fonte de estresse, insatisfação e de tensão no ambiente de trabalho, tendo-se em vista que o controle sobre o trabalho, está relacionado ao uso de habilidades no trabalho e, a autoridade de decisão sobre e no trabalho. ${ }^{3}$ Dessa forma, o 
controle remete a autonomia e à capacidade e liberdade criativa e participativa no trabalho.

Também os DPM estiveram associados ao menor grau de controle no trabalho $(\mathrm{OR}=1,64$ IC $95 \%=1,00-2,65 \mathrm{p}=0,037)$. Ou seja, os trabalhadores com baixo controle no trabalho apresentaram chances 1,67 vezes maior de desenvolver DPM que aqueles com alto controle no trabalho. Este achado é consistente com outros estudos. ${ }^{11-16}$ Esses resultados contribuem para a idéia de que pouca autonomia na organização de atividades parece disponibilizar mais ao adoecimento mental. ${ }^{12}$

Algumas situações no cotidiano de trabalho da enfermagem conferem grandes demandas ao trabalhador. Em um estudo observaram-se intensa movimentação dos profissionais de enfermagem no interior do hospital ao longo de todo o turno de trabalho e a preocupação destes em executar suas atividades a tempo. ${ }^{5}$ Soma-se a isso a necessidade de mover ou levantar cargas pesadas, a realização de esforço físico, atividades rápidas e contínuas e a necessidade de manter o corpo, a cabeça ou os braços em posições fisicamente incômodas por longos períodos. Sob esse prisma, evidenciou-se que quanto maior a demanda física, maior a prevalência de agravos à saúde mental. ${ }^{11}$

Este estudo revela que há prevalência de DPM entre os trabalhadores de enfermagem investigados, podendo essa ser associada a diferentes variáveis. O confrontamento destes dados com outros já encontrados na literatura, pode oportunizar a abertura para importantes reflexões, apontando medidas cabíveis no sentido de buscarem-se melhorias nas condições de trabalho destes sujeitos.

\section{CONSIDERAÇÕES FINAIS}

Com base na prevalência de DPM detectada e mantida após ajustes por fatores de confundimento entre trabalhadores com alta exigência no trabalho, este estudo corrobora com conhecimentos em relação aos efeitos danosos do trabalho em alta exigência à saúde mental dos trabalhadores.

Por isso, considera-se fundamental capacitar os trabalhadores dos serviços de saúde, quanto aos sinais e sintomas dos distúrbios psíquicos, para que considerem a importância da situação de trabalho como um dos fatores determinantes no processo saúde/doença. Desse modo, alerta-se para a necessidade de desenvolver ações interinstitucionais e multidisciplinares em saúde mental e trabalho. ${ }^{12}$ Além, da necessidade de uma gestão organizacional participativa, que inclua os traba- lhadores no processo de mudanças e melhorias do ambiente laboral. Somadas as medidas de promoção da saúde do trabalhador, ressalta-se o foco de atenção para a inclusão de medidas profiláticas que tenham início nos cursos profissionalizantes e nos de formação superior.

\section{REFERÊNCIAS}

1. Dejours C, Abdoucheli E, Jayet C. Psicodinâmica do trabalho: contribuições da Escola Dejouriana à análise da relação prazer, sofrimento e trabalho. São Paulo (SP): Atlas; 1994.

2. Pitta A. Hospital dor e morte como ofício. $2^{\mathrm{a}}$ ed. São Paulo (SP): Hucitec; 1991.

3. Karasek RA, Theörell T. Healthy work-stress, productivity, and the reconstruction of working life. New York (US): Basic Books; 1990.

4. Coffey M. Stress and burnout in forensic community mental health nurses: an investigation of its causes and effects. J Psychiatr Ment Health Nurs. 1999 Dec; 6(6):433-43.

5. Elias MA, Navarro VL. A relação entre o trabalho, a saúde e as condições de vida: negatividade e positividade no trabalho das profissionais de enfermagem de um hospital escola. Rev Latino-am Enfermagem. 2006 Jul-Ago; 14(4):517-25.

6. Martins-Nogueira, LA. Saúde Mental dos profissionais da saúde. Rev Bras Med Trab. 2003 Jul-Set; 1(1):56-68.

7. Coffey M, Colemann M. The relationship between support and stress in forensic community mental health nursing. J Adv Nurs. 2001 May; 34(3):397-407.

8. Araújo TM, Aquino E, Menezes G, Santos CO, Aguiar L. Aspectos Psicossociais do trabalho e distúrbios psíquicos entre trabalhadores da enfermagem. Rev Saúde Pública 2003 Ago; 37(4):24-33.

9. Amaral TRA. Dimensões psicossociais do trabalho da enfermagem e os distúrbios psíquicos menores em unidades críticas [dissertação]. Florianópolis (SC): Universidade Federal de Santa Catariana. Programa de Pós-Graduação em Enfermagem; 2006.

10. Silva BM, Lima FRF, Farias, FSAB, Campos ACS. Jornada de trabalho: fator que interfere na qualidade da assistência de enfermagem. Texto Contexto Enferm. 2006 Jul-Set; 15(3):442-8.

11. Silva JLL. Estresse e transtornos mentais comuns em trabalhadores de enfermagem [dissertação]. Rio de janeiro (RJ): Universidade Estadual do Rio de Janeiro. Programa de Pós-Graduação em Enfermagem; 2007.

12. Glina DMR, Rocha LE, Batista ML, Mendonça MGV. Saúde mental e trabalho: uma reflexão sobre o nexo com o trabalho e o diagnóstico, com base na prática. Cad Saúde Pública. 2001 Mai-Jun; 17(3):607-16. 
13. Cerchiari EAN, Caetano D, Faccenda O. Prevalência de Transtornos Mentais Menores em Estudantes Universitários. Estud Psicol (Natal). 2005 Set-Dez; 10(3):413-20.

14. Maragno L, Goldbaum M, Gianini RJ, Novaes HMD, César CLG. Prevalência de transtornos mentais comuns em populações atendidas pelo Programa Saúde da Família (QUALIS) no Município de São Paulo, Brasil. Cad Saúde Pública. 2006 Ago; 22 (8):1639-48.

15. Karasek RA. Job Content Questionnaire and user's guide [on line]. University of Massachusets; 1985 [acesso em 2007 Jun 10]. Disponível em: http:// www.jcqcenter.org

16. Araújo TM. Trabalho e distúrbios psíquicos em mulheres trabalhadoras de enfermagem. [tese]. Salvador (BA): Universidade Federal da Bahia. Programa de Pós-Graduação em Saúde Coletiva; 1999.
17. Mari JJ, Williams, P. A validity study of a psychiatric screening questionnaire (SQR-20) in primary care in the city of São Paulo. Br J Psychiatry. 1986 Jan; 148:23-6.

18. Sobrinho CLN, Carvalho FM, Bonfim TAS, Cirino CAS, Ferreira IS. Condições de trabalho e saúde mental dos médicos de Salvador, Bahia. Cad Saúde Pública. 2006 Jan; 22(1):131-40.

19. Souza MFM, Silva GR. Risco de distúrbios psiquiátricos menores em área metropolitana na região Sudeste do Brasil. Rev Saúde Pública. 1998 Fev; 32(1):50-8.

20. Batista AAV, Vieira MJ, Cardoso NCS, Carvalho GRP. Fatores de motivação e insatisfação no trabalho do enfermeiro. Rev Esc Enferm USP. 2005 Mar; 39(1):85-91. 